\title{
Nanoscale Chromatin Imaging and Analysis (nano-ChIA) Platform Bridges 4-D Chromatin Organization with Molecular Function
}

Yue Li, Adam Eshein, Ranya Virk, Aya Eid, David VanDerway, Jane Frederick, Reiner Bleher, Igal Szleifer, Vinayak Dravid and Vadim Backman

Northwestern University, Evanston, Illinois, United States

The dynamic, three-dimensional chromatin organization plays an important role in regulating a vast number of cellular processes, including cell-type-specific gene expression and lineage commitment (1-3). Large-scale alterations in chromatin structure are associated with cancer, numerous neurological and autoimmune disorders, and other complex diseases (4, 5). Extending across multiple length-scales - from the nucleosome to higher-order three-dimensional structures - chromatin is a dynamic system which evolves throughout the lifetime of a cell. However, the precise conformation of chromatin and its relationship with transcription, a direct determinant of cellular phenotype, remains contested.

A number of methods have been developed to analyze 3D chromatin organization, including chromatin conformation capture (e.g. Hi-C), neutron scattering, soft X-ray tom ography (SXT), super-resolution microscopy, partial wave spectroscopic (PWS) microscopy, and more recently developed chromEMT, which combines DNA-specific DNA labeling and transmission electron tomography,. These techniques have provided critical insights into the principles of 3D chromatin structure, but they have fundamental limitations rooted the philosophy of experimental designs, characterization resolution, or sample preparation protocols $(6)$. Because no individual technique can fully elucidate the chromatin organization and its relation to molecular function at all length and time scales (7), it is necessary to create an ensemble combining complementary techniques. To meet these requirements, we have developed the Nanoscale Chromatin Imaging and Analysis (nano-ChIA) platform, which includes chromatin scanning transmission electron microscopy (ChromSTEM), PWS, and stochastic optical reconstruction microscopy (STORM) (Fig. 1A-1D). As a demonstration of the capability of nano-ChIA, we sought to investigate the chromatin higher-order structure and its relation to gene transcription.

Utilizing nano-ChIA, we observed that chromatin is localized into spatially separable packing domains (PDs), with an average diameter of around $200 \mathrm{~nm}$ and an internal fractal structure. First, we employed the ChromSTEM module to resolve the 3D chromatin organization of A549 human lung adenocarcinoma cells $(\mathrm{N}=4)$ at sub-3 nm nominal resolution (Fig. 1E-1G). We further quantified the chromatin packing using mass-scaling analysis, defined as the relationship between the radius of a sphere and the total chromatin mass encapsulated by the sphere. From polymer physics, the mass-scaling relationship of the chromatin follows a power-law relationship, with a power-exponent $D$, which is indicative of chromatin packing conformation. From mass-scaling, we did observe such regional power-law behavior (Fig. 1H), as well as randomly distributed chromatin PDs with similar $D$ value within the domains (Fig. 1I) We further employed PWS to inspect the chromatin packing scaling distribution across the entire nucleus and confirm the presence of packing domains in live cells. In agreement with ChromSTEM, PWS analysis also identified spatially separable domains characterized by similar D within the domains (Fig. 1J-1K).

Next, we leveraged nano-ChIA to explore the relationship between chromatin packing and gene transcription. Specifically, we employed STORM-PWS module to localize regions of active gene transcription by imaging active RNA Polymerase-II (Pol-II) with STORM and measured the surrounding chromatin packing scaling using PWS (Fig. 2A-2B). We observed a non-monotonic relationship between $D$ and expression rate, which agrees with the prediction by a chromatin packing macromolecular crowing 
(CPMC) model we developed previously (Fig. 2C) (8). Notably, we found that Pol-II density is associated with observed PDs, with pockets of high Pol-II density forming around the periphery of PDs or groups of PDs (Fig.2D). In order to test if PDs are formed, at least in part, by active gene transcription, we performed a perturbation study to halt transcriptional elongation in BJ fibroblast cells by treatment with Actinomycin D (Act-D) (9). After Act-D treatment, we continuously captured PWS images for ten minutes to evaluate the real-time effect of transcriptional inhibition on chromatin structure from the level of PDs to the scale of the whole nucleus (Fig. 2E). At the level of the entire nucleus, we observed that treatment with Act-D produces a rapid decrease in average chromatin packing scaling $D$ across the cell population (Fig. 2F). Importantly, the abrogation of transcription did not eliminate the PD structure of chromatin (Fig. 2E right panel, $2 \mathrm{~F}, 2 \mathrm{G}$ ). Our findings are consistent with previous work showing that chromatin structure is stabilized by transcriptional elongation (10). Furthermore, this result supports the hypothesis that the process of active gene transcription affects supranucleosomal chromatin organization but is not its sole determinant.

In summary, each facet of nano-ChIA interrogates distinct aspects of chromatin architecture in the context of molecular processes: ChromSTEM for DNA density and the spatial conformation of the chromatin chains, PWS for label-free, dynamic measurements of the statistical properties of the chromatin conformation in live cells, and STORM for in situimaging of molecular functions with nanoscale resolution. Through nano-ChIA, we identified chromatin forms distinctive PDs with varying size and packing conformation throughout the nucleus, and the packing properties within the domain exhibit a complex bi-directional relationship with active gene transcription. Further studies employing the nanoChIA platform have the potential to provide a more coherent picture of chromatin structure and its relation to molecular function. 


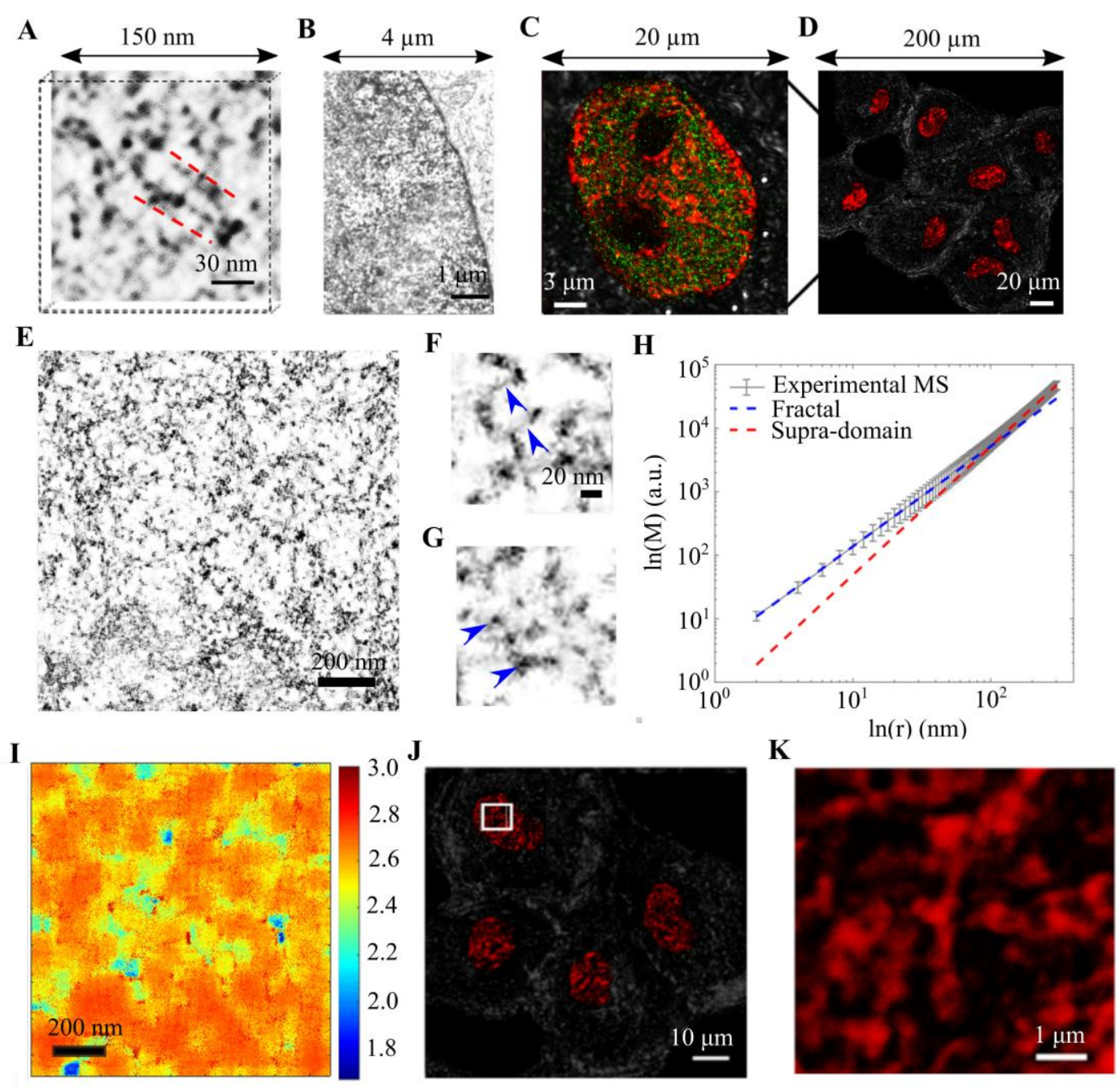

Figure 1. Chromatin packing domains (PDs) observed by nanoscale Chromatin Imaging and Nanoscale Analysis (nano-ChIA) platform. (A) ChromSTEM HAADF tomography characterizes the 3D chromatin structure of an A549 cell (contrast inverted). The inverted image contrast in ChromSTEM HAADF tomography is inversely proportional to the local DNA density: as the electrons encounter a higher density of DNA along their trajectory, the image contrast appears darker. Individual nucleosomes and linker DNA are resolved at sub 3-nm spatial resolution. Scale bar: $30 \mathrm{~nm}$. (B) ChromTEM imaging of a bJ cell nucleus on a $50 \mathrm{~nm}$ resin section prepared by ChromEM staining. Similar to ChromSTEM, ChromTEM also maps the DNA distribution, but the image contrast follows Beer's law. Scale bar: $1 \mu \mathrm{m}$. (C) Coregistered PWS and STORM imaging of chromatin packing scaling D (pseudo color: red) within chromatin packing domains and active RNA polymerase II (green) of an M248 cell nucleus. Scale bar: $3 \mu \mathrm{m}$. (D) Label-free PWS images of live A549 cells. The pseudo color again represents the chromatin packing scaling D. Scale bar: $20 \mu \mathrm{m}$. (E) A $2.9 \mathrm{~nm}$ thick virtual 2D slice from ChromSTEM HAADF tomography reconstruction of the chromatin from an A549 cell nucleus (contrast inverted). Scale bar: $200 \mathrm{~nm}$. (F-G) High-resolution tomography reveals find chromatin structures such as linker DNA (blue arrows in F) and individual nucleosomes (blue arrows in G). Scale bar: $20 \mathrm{~nm}$. (H) Average mass scaling of the chromatin packing in $\log -\log$ scale shows a packing domain regime with an internal fractal structure. Within individual PDs, 
mass and physical space occupied by chromatin follow a power-law relationship (linear in the log-log plot). The dashed lines depict the linear regression fit within each regime. (I) Mapping of chromatin packing scaling D of an A549 cell. The chromatin self-organizes into packing domains (PDs) with similar packing scaling. Scale bar: $200 \mathrm{~nm}$. (J) Mapping of chromatin packing scaling D of the whole cell by PWS. Scale bar: $10 \mu \mathrm{m}$. (K) Magnified PWS mapping of D of the region highlighted by the white square in $(\mathrm{J})$. Each cluster represents a diffraction-limited observation of PDs. Scale bar: $1 \mu \mathrm{m}$.
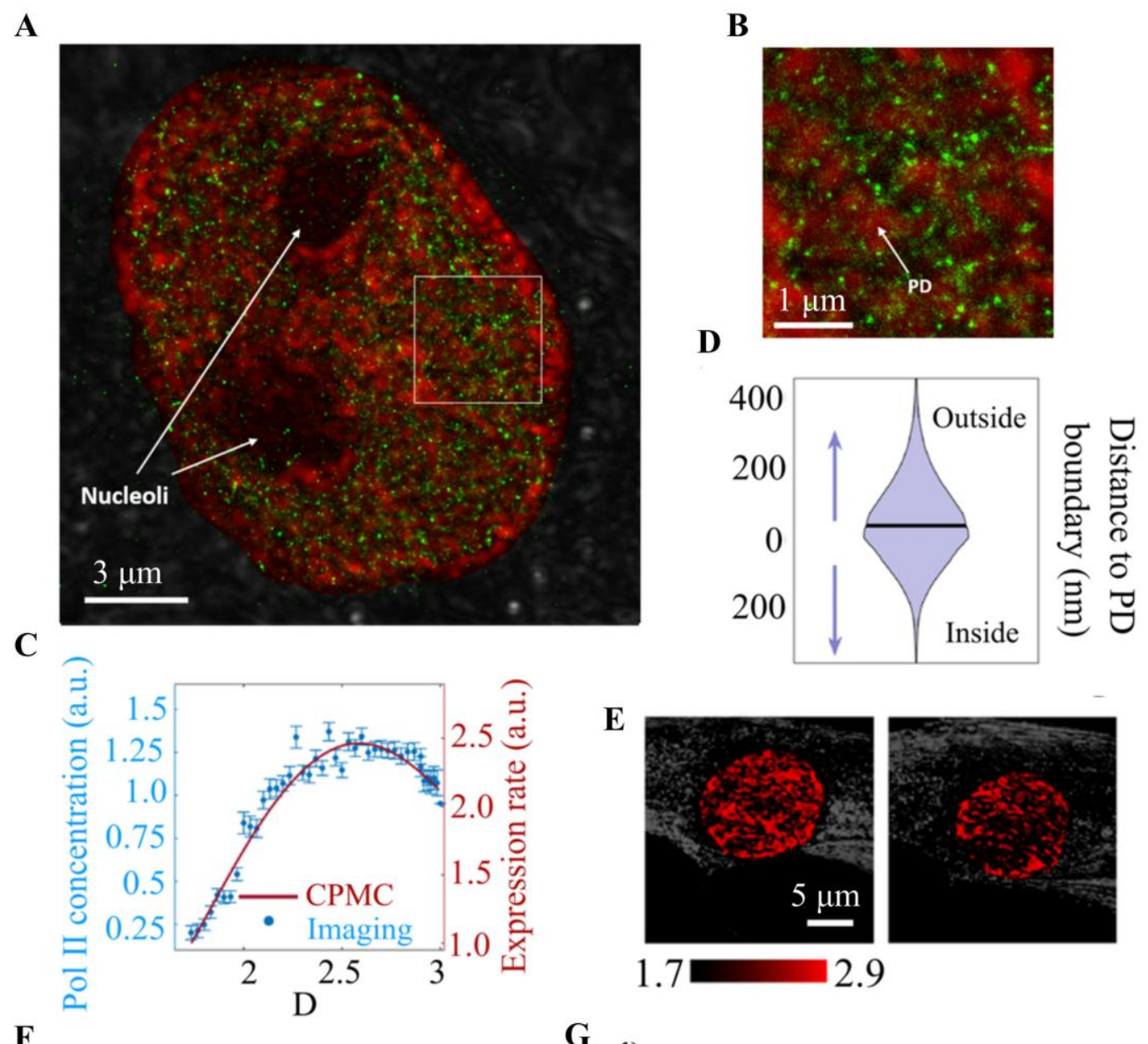

$\mathbf{F}$
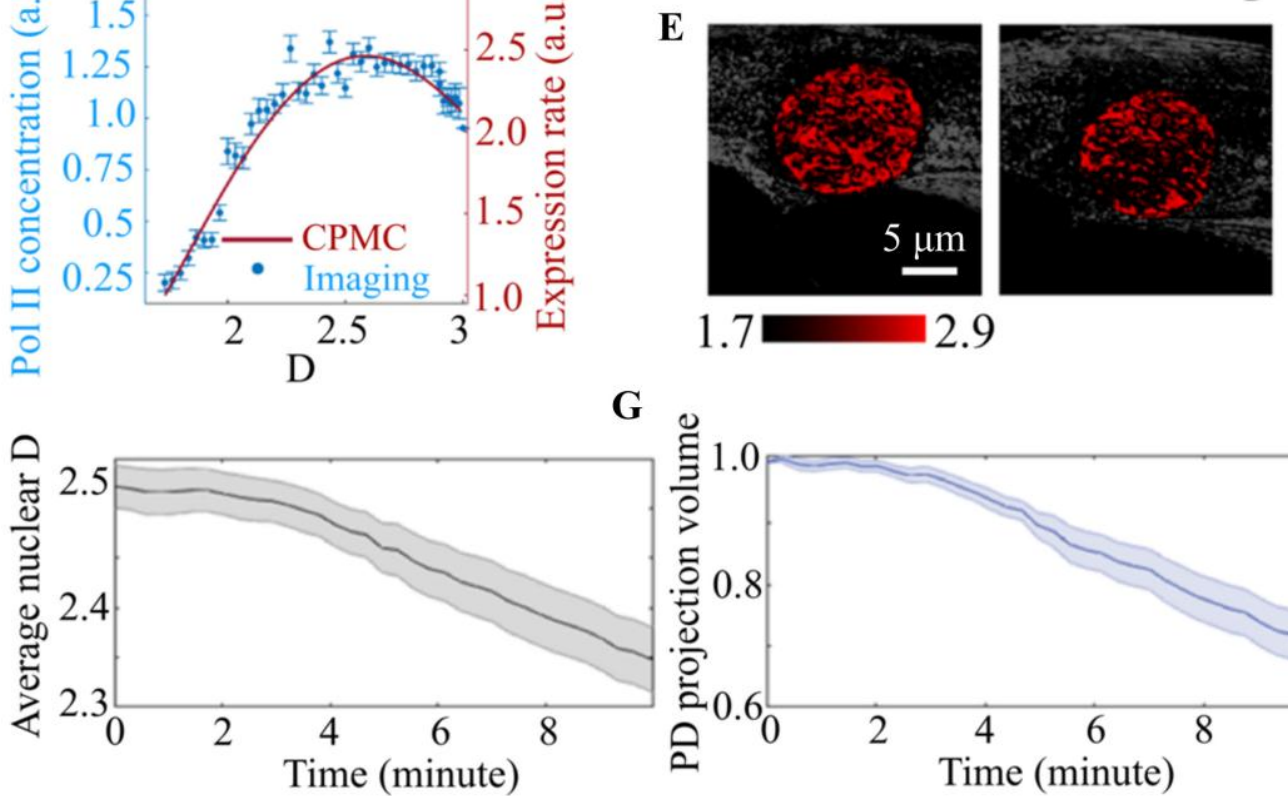

G

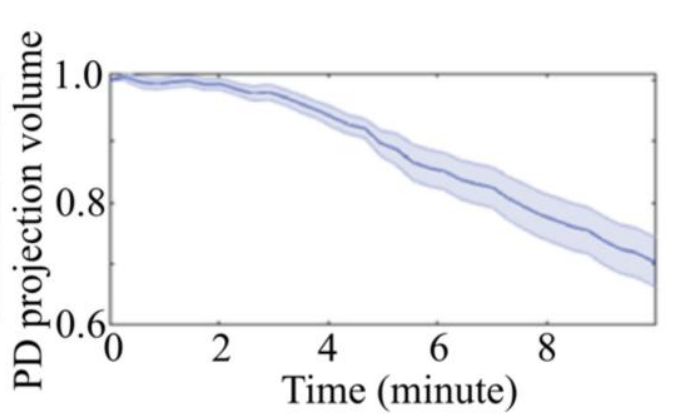

Figure 2. Nano-ChIA platform investigates the relationship between chromatin structure and transcription. (A) A STORM image of an M248 ovarian cancer cell labeled with RNA polymerase II (green) overlaid on top of chromatin packing scaling D measured by PWS (red). Scale bar: $3 \mu \mathrm{m}$. (B) A magnified view of the white square in $(\mathrm{A})$. Scale bar: $1 \mu \mathrm{m}$. (C) The relationship between $\mathrm{D}$ and the local 
concentration of RNA polymerase II (gene expression level) compared with one realization of the CPMC model. (D) A violin plot shows the distribution of distances between enriched RNA polymerase II regions and their nearest packing domain group. The plot shows that RNA polymerase II tends to distribute around the boundary of packing domains. (E) PWS imaging of a live BJ fibroblast cell during Act-D treatment. The pseudo color is coded by D values inside the nuclei. Scale bar: $5 \mu \mathrm{m}$. (F) After transcriptional elongation is halted with Act-D, average nuclear chromatin packing scaling decreased steadily within minutes. $(\mathrm{G})$ The change in the volume fraction of the nucleus containing packing domains as measured by PWS.

\section{References}

1. J. E. Phillips-Cremins et al., Architectural protein subclasses shape 3D organization of genomes during lineage commitment. Cell 153, 1281-1295 (2013).

2. E. J. Clowney et al., Nuclear aggregation of olfactory receptor genes governs their monogenic expression. Cell 151, 724-737 (2012).

3. J. S. Becker et al., Genomic and Proteomic Resolution of Heterochromatin and Its Restriction of Alternate Fate Genes. Mol Cell 68, 1023-1037 e1015 (2017).

4. P. C. Taberlay et al., Three-dimensional disorganization of the cancer genome occurs coincident with long-range genetic and epigenetic alterations. Genome Res 26, 719-731 (2016).

5. P. Panikker et al., Restoring Tip60 HAT/HDAC2 Balance in the Neurodegenerative Brain Relieves Epigenetic Transcriptional Repression and Reinstates Cognition. J Neurosci 38, 4569-4583 (2018).

6. Y. Li et al., Quantifying Three-dimensional Chromatin Organization Utilizing Scanning Transmission Electron Microscopy: ChromSTEM. bioRxiv, 636209 (2019).

7. M. Cremer, T. Cremer, Nuclear compartmentalization, dynamics, and function of regulatory DNA sequences. Genes, Chromosomes and Cancer 58, 427-436 (2019).

8. R. K. Virk et al., Disordered chromatin packing regulates phenotypic plasticity. Science Advances 6 , eaax6232 (2020).

9. X. Darzacq et al., In vivo dynamics of RNA polymerase II transcription. Nature Structural \&Amp; Molecular Biology 14, 796 (2007).

10. T. Nozaki et al., Dynamic Organization of Chromatin Domains Revealed by Super-Resolution LiveCell Imaging. Mol Cell 67, 282-293 e287 (2017). 